\title{
Image Converter and Intensifier Investigations on Luminous Fronts (Ionizing Waves) in Nitrogen Discharges in a Homogeneous Field
}

\author{
J. KoppITZ \\ Institut für Angewandte Physik der Universität Hamburg \\ (Z. Naturforsch. 26 a, 700—706 [1971] ; received 23 December 1970)
}

\begin{abstract}
In this paper optical space and time resolved measurements on the later development of Kanal (streamer) and Townsend discharges in nitrogen are treated. In both discharges fast luminous fronts (the so called ionizing waves) arise: in the streamer discharge between the primary streamer stage and the spark stage, and in the Townsend discharge during the transition to the glow.

For the streamer discharge new experimental results are given, especially at high overvoltages. In the case of the Townsend discharge the development of an idealized, nearly one dimensional discharge is treated. The experimental results are in good agreement with theoretical considerations. In contrast to this idealized discharge often strong constrictions are found during the development to the glow, so that the discharge becomes nearly filamentary. The reasons for this behaviour are discussed and the way to avoid it.
\end{abstract}

\section{Introduction}

"Ionizing waves" in gas discharges are fast moving regions of high ionisation rates combined with higher luminosity. They can be observed by photomultiplier or by streak photograph methods. The growth in the degree of ionisation produced by them leads to a steep rise of the discharge current. Ionizing waves are found in various types of gas discharges, e. g. in Kanal (or streamer) discharges between the first streamer stages and the formation of the spark, or in Townsend discharges during the transition to the glow. The electrical field may be homogeneous or inhomogeneous ${ }^{1}$.

In this paper optical measurements on ionizing waves are described. Two types of discharges are treated, Kanal and Townsend mechanism ${ }^{2}$ in a homogeneous field. The gap distance was $2 \mathrm{~cm}$, the diameter of the electrodes $14 \mathrm{~cm}$. The measurements were done in pure nitrogen because of its high photon efficiency. The applied electrical field could be pulsed with a rise time of $20 \mathrm{nsec}$. The primary electrons were produced by a flash lamp either over the whole cathode or in a small area of about $0.25 \mathrm{~mm}^{2}$. For the optical investigations an image converter followed by an image intensifier was used because a resolution in time and space was needed. The image converter could be driven as a fast

Reprints request to J. Koppitz, Institut für Angewandte Physik der Universität Hamburg, D-2000 Hamburg 36, Jungiusstraße 11 .

1 W. P. Winn, J. Appl. Phys. 38, 783 [1967].

2 H. Raether, El. Avalanches and Breakdown in Gases, Butterworths, London 1964. shutter with a minimum exposure time of $3 \mathrm{nsec}$ and as a streak camera with a time resolution better than one nanosecond.

\section{Kanal Mechanism}

The mechanism requires high overvoltage conditions. For $\mathrm{N}_{2}$ at $p \sim 10^{2}$ Torr $\Delta U / U_{0}$ must be greater than $17 \%$ which makes $\ln n_{0}+\alpha d>(\alpha d)_{\mathrm{cr}} \approx 20$ ( $\Delta U=$ overvoltage, $U_{0}=$ stat. breakdown voltage, $\alpha=$ Townsend's first coefficient, $d=$ gap distance, $n_{0}=$ number of starting electrons within the diffusion radius of the avalanche). The primary avalanche at a position $X_{\text {cr }}$ transforms into an anode directed and a cathode directed streamer under the influence of its own space charge. The phenomenology and the basic theory of the mechanism are given by RAETHER $^{2}$, WAGNER ${ }^{3,4}$ and ReIninghaUs ${ }^{5}$. It has been shown by $\mathrm{WAGNER}^{4}$ and KoppITZ ${ }^{6}$ that fast luminous fronts are generated moving in both directions through the gap when the streamers arrive at the electrodes. Then a glow-like stage follows for a few ten nanoseconds. It ends with the thermalisation of the gas which starts near $X_{\mathrm{cr}}{ }^{6,7,3}$. The final development is the well known hydrodynamic expansion of the heated gas (e. g. ${ }^{8}$ ). The average radius of the streamer discharge is of the order of the avalanche diffusion radius till the formation of

\footnotetext{
${ }^{3}$ K. H. Wagner, Z. Phys. 189, 465 [1966].

${ }^{4}$ K. H. Wagner, Z. Phys. 204, 177 [1967].

5 W. Reininghaus, to be published.

6 J. Koppitz, Z. Naturforsch. 22 a, 1089 [1967].

7 H. ThOlL, Z. Naturforsch. 22 a, 1068 [1967].

8 H. Tholl, Z. Naturforsch. 25 a, 420 [1970].
} 
the hot spark. At a pressure of about $10^{2}$ Torr the radius is about $0.5 \mathrm{~mm}$, thus small against the gap distance of $2 \mathrm{~cm}$.

The later stage of Kanal mechanism not well known today is that of the fast luminous waves. Here some new results on this stage shall be reported. The measurements were made in nitrogen at a pressure of 300 Torr. About $10^{3}$ primary electrons were started in the midth of the cathode surface. An overvoltage of $18 \%$ to $60 \%$ was applied to the gap. An example of the later stage of the streamer development and the following ionisation waves for higher overvoltages is shown in the streak photograph of Fig. 1 (35\% overvoltage). In the
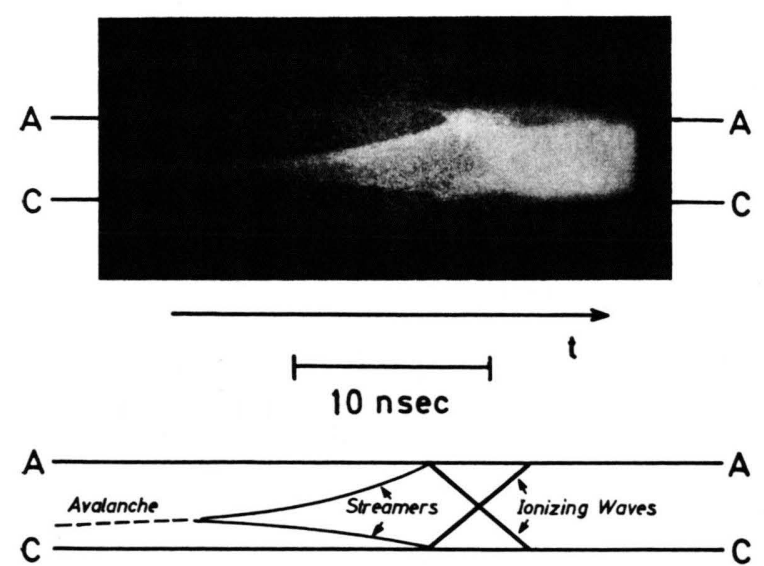

Fig. 1. Streak photograph of a streamer discharge at high overvoltage. Upper part : original photograph; lower part: schematic diagram. Conditions: $\mathrm{N}_{2}$ at 300 Torr; $d=2 \mathrm{~cm} ; \Delta U / U_{0}$ $=35 \% ; U_{0}=25.6 \mathrm{kV}\left(E_{0} / p=42.7 \mathrm{~V} / \mathrm{cm}\right.$ Torr $) ; n_{0} \sim 10^{3}$. The anode and cathode directed streamers and the fast ionizing waves crossing one another are visible (A: anode, C: cathode).

same figure the development is drawn schematically. The investigations lead to the following results:

a) At overvoltages of more than $25 \%$ the development of the anode and cathode directed streamers becomes more and more symmetrical concerning velocity (Fig. 1, see also ${ }^{9}$ ). The more complicated structure of the cathode directed streamer as reported by WAGNER ${ }^{3}$ and REININGHAUS ${ }^{5}$ for low overvoltages with a slow stage and a later brighter and faster stage disappears.

b) The initial velocities of both streamers become comparable at higher overvoltages. Further on it is observed that the streamer velocity increases on the way to the electrodes.

9 K. Möstl and U. Trмm, Z. Phys. 209, 60 [1968]. c) Each streamer when arriving at an electrode starts a very fast front toward the opposite electrode, the "ionizing wave". (These phenomena are no more called "streamers" since the ionizing waves develop inside the plasma left behind the streamers. It is still an open question how far both events can be described theoretically by the same formalism.) The velocity of the cathode directed luminous front $\left(v_{\downarrow}\right)$ strongly increases with the applied field while the anode directed front $\left(v_{\uparrow}\right)$ becomes slower (Fig. 2). At high overvoltages both fronts

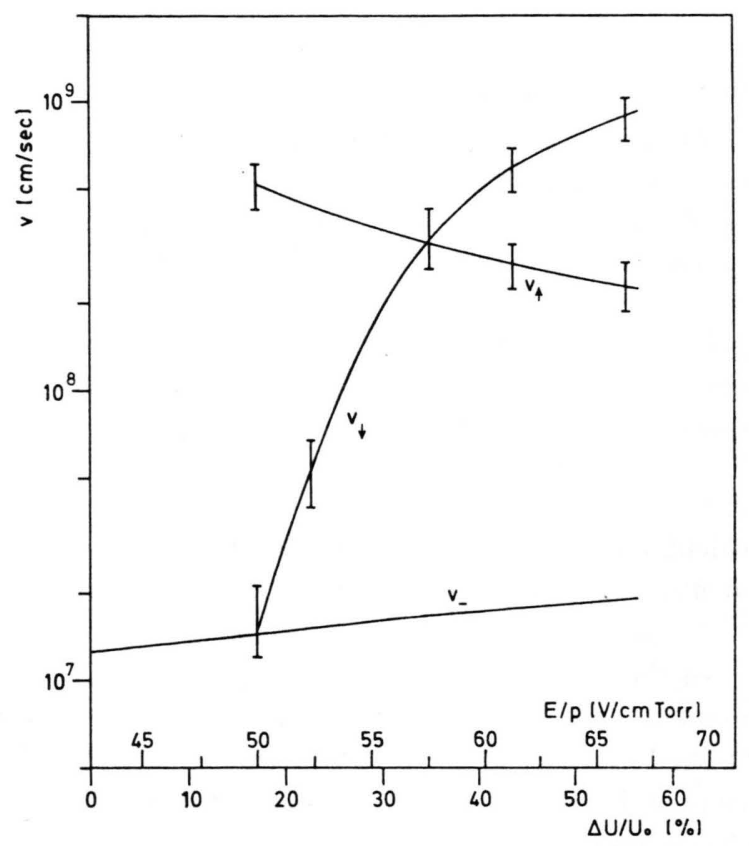

Fig. 2. Dependence of the velocities of the ionizing waves in Fig. 1 on the overvoltage. Anode directed wave: $v_{\uparrow}$; cathode directed wave: $v_{\downarrow}$; electron drift velocity (for comparison) : $v_{-}$. The conditions are the same as in Fig. 1 .

move at nearly constant velocity from one electrode to the other and do not change it when crossing one another. Both fronts generate still faster new ones moving in the opposite direction respectively when they arrive at the electrodes but the contrast of the photographic film is often too low to deduce detailed features.

\section{Townsend Mechanism}

\section{Introductory remarks}

When an overvoltage of only a few per cent or the static breakdown voltage is applied to the gap under the same experimental conditions as above 
and the density of the starting electrons is not too high (for high density see Schlumborm ${ }^{10}$ ) the primary avalanche is not influenced by its own space charge and the development of the discharge is governed by the generation mechanism (Townsend mechanism). Under the conditions described above the successors are produced by photo effect at the cathode. The time interval between two generations is about $\mathrm{T}-$. Due to the photoeffect the discharge spreads over the cathode area within few generations ${ }^{11}$ provided that the secondary electron emission of the cathode is constant over this area. Thus the discharge radius is usually larger than the gap distance.

After the first oscillations the average electron current becomes theoretically constant for $\mu=1$ and rises exponentially for $\mu>1$ (But in reality the space charge produces an exponential rise also for $\mu=1)$. It is well known that after a number of generations a steep overexponential rise of the current begins due to the influence of the positive space charge accumulated in the gap (e. g. ${ }^{12}$ ). The first stages of this development, when the field distortions are still small (a few per cent) were calculated in quantitative agreement with experiments, e.g. by Schlumborm (see in ${ }^{2}$ ) and HESSENAUER ${ }^{11}$.

In the following stages of the discharge development the transition to the glow takes place. It is studied by space and time resolving optical methods. Here a high field region is generated in the discharge due to the space charge of the positive ions. This moves fast through the gap, and the movement is detectable as a luminous front by optical methods. The first measurements of that kind were made by DorAN ${ }^{13}$ at this institute at a fixed overvoltage of $7.56 \%$.

\section{Results}

In the next sections new experimental results are reported. First the development of a discharge is described whose diameter is large compared with the gap distance and remains constant till the glow stage ("one dimensional" discharge). The experimental results are compared with theoretical considerations. Then discharges are treated in which a strong radial contraction takes place during the

10 H. Schlumbohm, Z. Phys. 170, 233 [1962].

11 H. Hessenauer, Dissertation, to be published. development to the glow. The reasons for the contraction are discussed and the way to avoid it.

The temporal development of the light distribution in a broad, nearly one dimensional Townsend discharge for low overvoltages $\left(\Delta U / U_{0}<10 \%\right)$ is demonstrated by streak photographs (Fig. 3) and
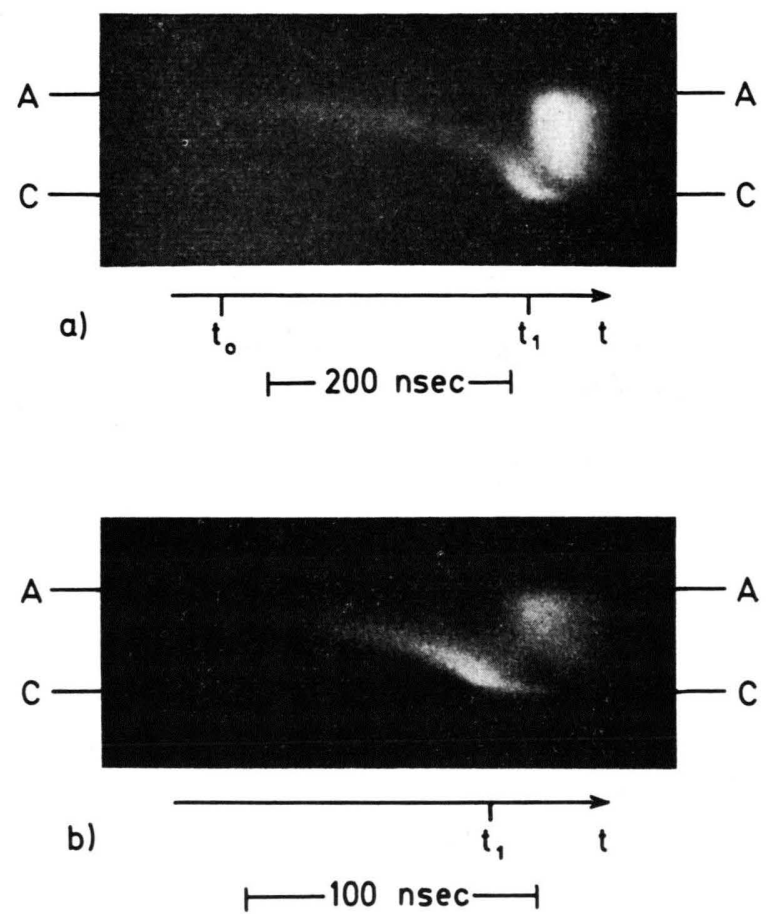

Fig. 3. Streak photographs of the development of a Townsend discharge with large diameter into the glow stage; overvoltage $\Delta U / U_{0}=1 \%$, the other conditions as in Fig. 1. At the time $t_{0}$ (discharge current density $j \sim 0.5 \mathrm{~mA} / \mathrm{cm}^{2}$ ) the first luminous front starts from the anode and arrives at the cathode at $t_{1}=t_{0}+250 \mathrm{nsec}$. Then a second luminous front starts from the anode resulting in the glow stage (A: anode, $\mathrm{C}$ : cathode).

by a series of shutter photographs (Fig. 4). The influence of the overvoltage is discussed later. In Fig. $3 \mathrm{a}$ one can see that at a time $t_{0}$ a luminous front starts from the anode and moves to the cathode with rising velocity within $\sim 250 \mathrm{nsec}$. The initial velocity is about $0.3 \cdot 10^{7} \mathrm{~cm} / \mathrm{sec}$, the final velocity $4.5 \cdot 10^{7} \mathrm{~cm} / \mathrm{sec}\left(v_{-}\right.$for a few per cent overvoltage is $\left.1.3 \cdot 10^{7} \mathrm{~cm} / \mathrm{sec}\right)$. In Fig. 3 it is seen that shortly after the arrival of the first front at the cathode $\left(t_{1}=t_{0}+250 \mathrm{nsec}\right)$ a new very fast front starts from the anode $\left(v \sim 10^{8} \mathrm{~cm} / \mathrm{sec}\right)$. Afterwards the brightness is uniform throughout the gap except

12 J. Pfaue, Z. Angew. Phys. 16, 15 [1963].

13 A. A. Doran, Z. Phys. 208, 427 [1968]. 


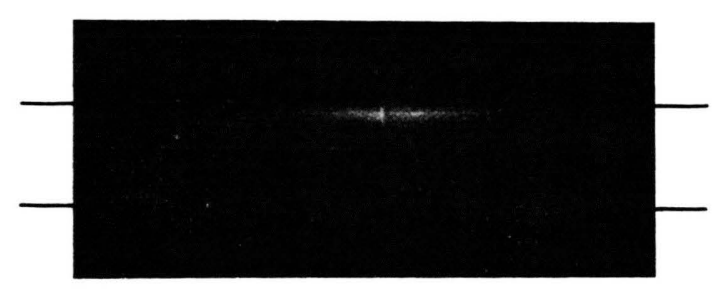

a) to

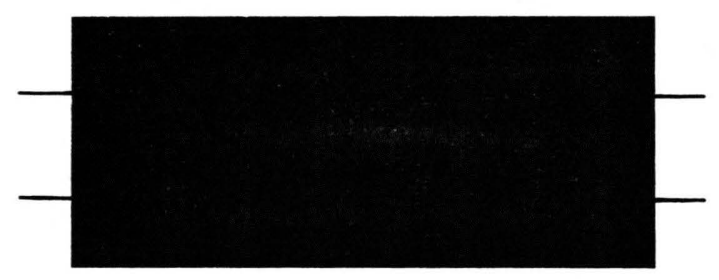

b) to+160nsec

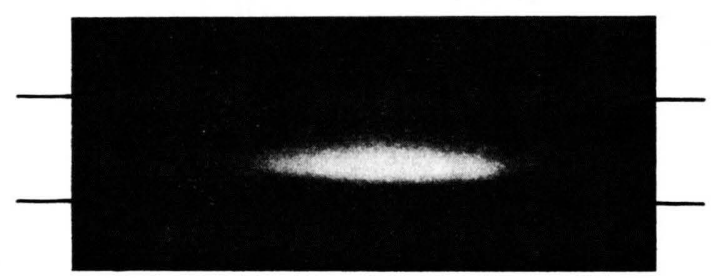

c) $t_{0}+235 \mathrm{nsec}$

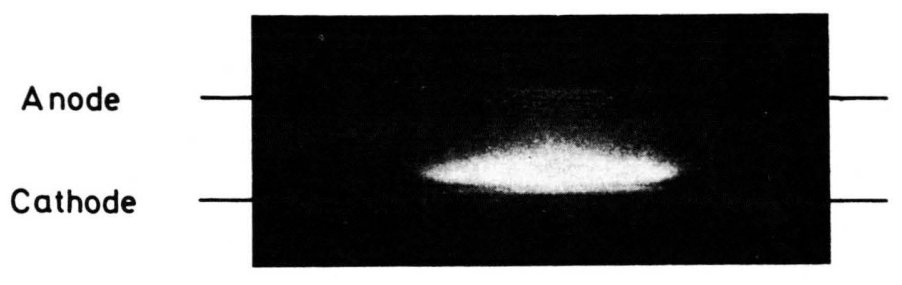

d) to $+245 \mathrm{nsec}$

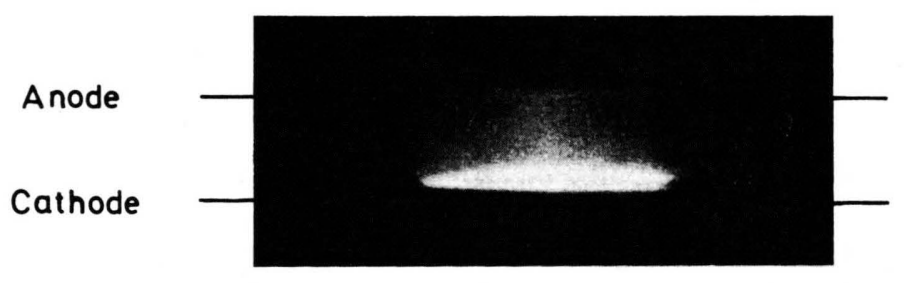

e) $t_{0}+250$ nsec $=t_{1}$

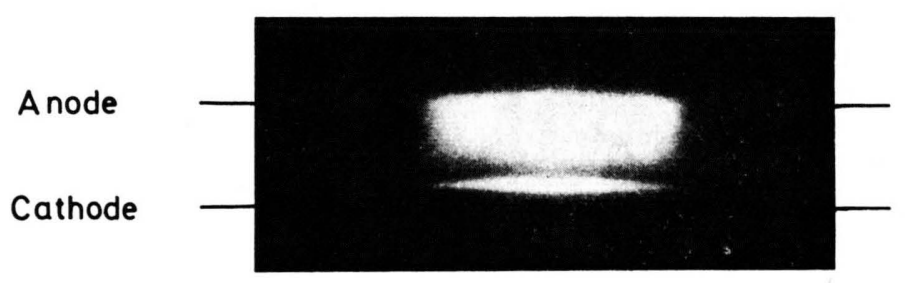

f) to +280 nsec

Fig. 4. Shutter photographs representing the development of the first luminous front in the large diameter Townsend discharge (a-e) and the final glow stage (f). Conditions as in Fig. 3. Fig. 4 a: integrated shutter photograph, shutter closed at $t_{0}$; Fig. $4 \mathrm{~b}-\mathrm{f}$ : exposure time 3 nsec. Discharge diameter $D \sim 5 \mathrm{~cm}$. $D$ stays constant during the development to the glow stage.

a small dark space near the cathode (quasi stable glow stage). The electrical current density was estimated $j \sim 0.5 \mathrm{~mA} / \mathrm{cm}^{2}$ at the very slow start of the first luminous front $\left(t_{0}\right)$. The time $t_{1}$ is marked in the current oscillograms as a dip, see Fig. 5 a.

The same development as in Fig. 3 is shown in a series of shutter photographs in Fig. 4. Fig. $4 \mathrm{a}$ is an integrated shutter photograph with the shutter closed at $t_{0}$. It is seen that until then a luminous layer with diameter $D \sim 5 \mathrm{~cm}$ is formed at the anode (gap distance $d=2 \mathrm{~cm}$ ). The movement of the first luminous front is illustrated by Fig. $4 \mathrm{~b}-\mathrm{e}$.

These photographs were taken at an exposure time of 3 nsec. The very short exposure time of the RCA 4449 A electron optical shutter was made possible by using an avalanche transistor pulse generator for the $300 \mathrm{~V}$ grid control pulses. As can be seen in Fig. $4 \mathrm{~b}-\mathrm{e}$ a thin luminous dis is moving to the cathode with nearly constant diameter and an estimated thickness of $1-2 \mathrm{~mm}$ (the optical device was driven at high opening ratio and the discharge diameter was comparable with the image distance). Fig. $4 \mathrm{f}$ is a $3 \mathrm{~ns}$ exposure time photograph of the final glow stage of the discharge taken 30 nsec after $t_{1}$.

From Fig. 4 one can conclude that the discharge diameter $D$ stays constant during all the development, $D \sim 5 \mathrm{~cm} . D$ depends mainly on the treatment of the cathode (see the final section of this paper). Considering that Fig. 4 is taken by side-on measurement the intensity is nearly constant over the discharge area. Thus the discharge can be regarded as a one dimensional discharge in a good approximation. 
This development of the first luminous front can be understood in the following way: During the initial stages of the Townsend discharge positive ions are generated mainly near the anode of the gap. The ions are accumulated and the field is diminished in the anode region and raised in the cathode region by the resulting space charge layer. The succeeding avalanches get a higher amplification in the high field region and produce new ions at the cathode side of the space charge layer. So the field distortion increases and the high field region is shifted toward the cathode. Since the maximum of ionisation and light emission is situated at the cathode end ${ }^{11}$ of the ion cloud this movement is detectable as a luminous front.

The negative space charge of the generated electrons can be neglected during the slow stage of the first front in the beginning of its development because the electrons move fast to the anode and vanish there. But when the front approaches the cathode it becomes so fast that the electrons cannot reach the anode and build up a negative space charge ${ }^{11,14}$. The field at the anode rises again and the second luminous front starts at the anode.

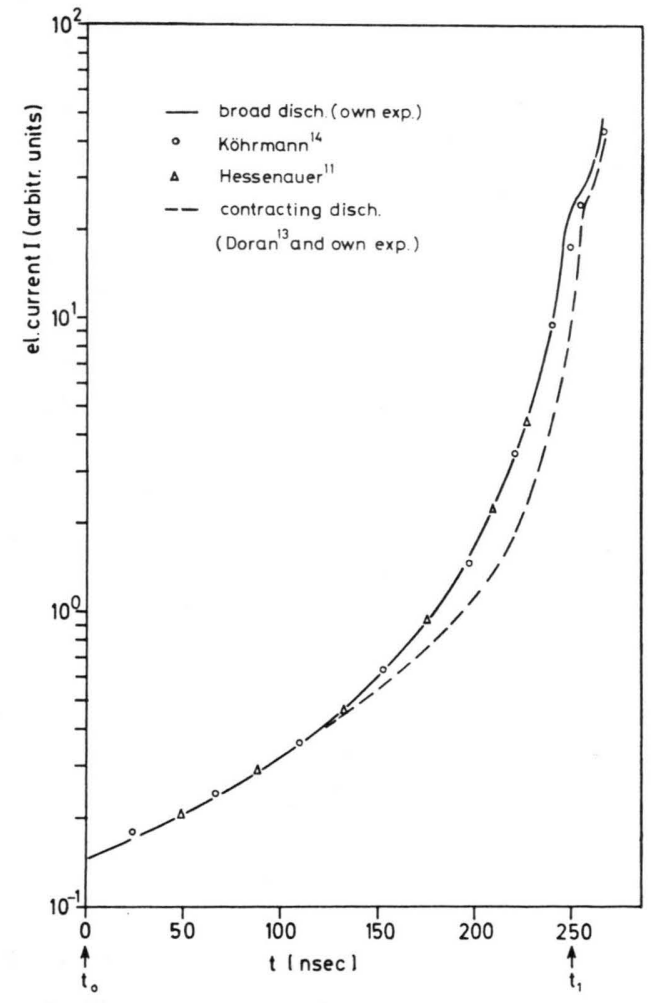

14 W. Köhrmann, Z. Naturforsch. 19 a, 926 [1964].

\section{Comparison with Theoretical Considerations}

The formation of the first high field region which moves to the cathode and is detectable by the luminous front was calculated by KÖHRMANN ${ }^{14}$ and Hessenauer ${ }^{11}$. The considerations of Köhrmann also include the second luminous front and the onset of the glow. Both authors assumed a one dimensional discharge (unlimited discharge area). Since the discharge shown in Fig. 4 fulfil this condition a comparison was tried between the experimental and theoretical results:

The total electrical current was measured over a small resistance with a fast oscilloscope. A comparison of the calculated and measured discharge current during the transition to the glow is shown in Fig. 5 a. Low overvoltage conditions were used $\left(\Delta U / U_{0} \approx 10^{-2}\right)$. Therefore such a high number of generations had crossed the gap before the first luminous front has started (in the experiment 20) that the initial current oscillations have already vanished. The calculations of Köhrmann for

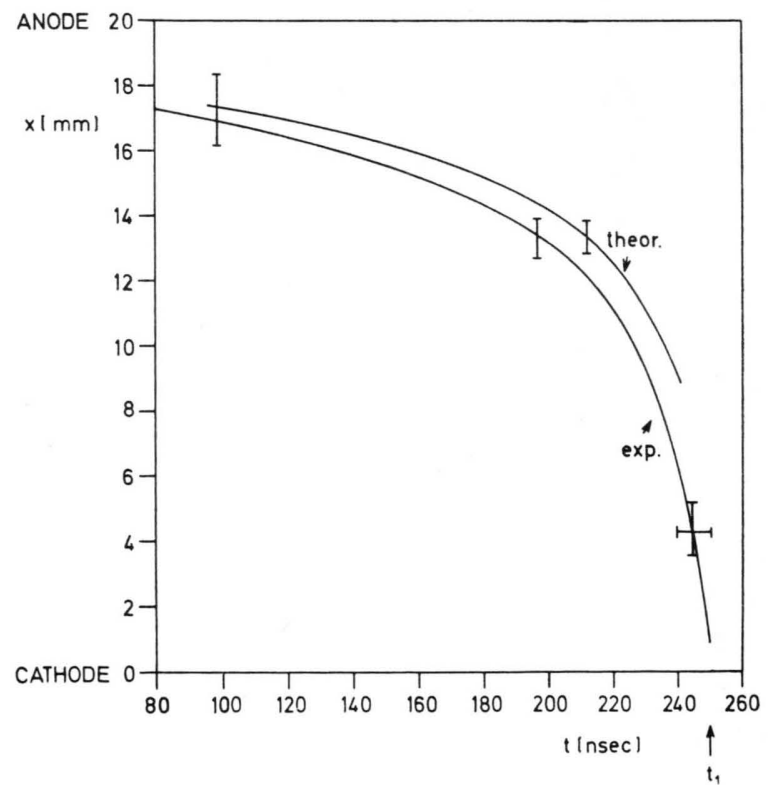

Fig. 5. Comparison between experimental and theoretical results of the transition of the Townsend discharge into the glow. Fig. 5 a shows the electrical current development (arbitrary units). The curves are fitted in time at the dip in the current curve $\left(t_{1}\right)$, and in the current amplitude at the first nearly exponential development $\left(t_{0}\right)$. (The dotted curve is concerned with discharges with a diameter diminishing from $t_{\mathbf{0}}$ to $t_{\mathbf{1}}$.) Fig. $5 \mathrm{~b}$ gives the position of the first luminous front as a function of time. The experimental values are taken from streak photographs. The theoretical values ${ }^{11}$ correspond to the maximum of the calculated light intensity. 
$\mathrm{H}_{2}$ were applied to $\mathrm{N}_{2}$; they are in good agreement with the calculations of HEssenauer ${ }^{11}$. For the dotted curve see the last section of this paper. The agreement between the calculations and our experimental results is good as Fig. 5 a shows.

In Fig. $5 \mathrm{~b}$ the temporal development of the first luminous front is shown. The experimental curve demonstrates the movement of the light intensity maximum visible in the streak photographs. The calculated values of HESSENAUER ${ }^{11}$ are the calculated positions of maximum light emission in the gap. Again the agreement between the measurements and the calculations is good.

\section{Influence of overvoltage}

In further experiments the influence of the applied overvoltage on the stage of the luminous waves was investigated. For overvoltages between $0 \%$ and about $10 \%$ no influence could be found. Only the number of generations changes which are required to accumulated enough space charge for the start of the luminous front (Fig. $4 \mathrm{a}$ ). This is in agreement with former calculations (e. g. ${ }^{14}$ ) which show that the "asymptotic" behaviour of the transient current of a Townsend discharge is independent on the initial conditions.

At overvoltages $\Delta U / U_{0}$ greater than $12 \%$ the transition to the glow takes place within the second or even the first generation. The experiments were done with $>10^{6}$ primary electrons started on the whole cathode area. Then the second or, at about $14 \%$ overvoltage, the first generation is visible as a luminous disc moving to the anode. During this development a second luminous front arises which moves to the cathode. No radial constriction can be found. The formative time (from the first electron to the glow stage) amounts only $1 \ldots 2 T_{-}$. The explanation of this type of discharge will be discussed in detail in a later publication.

\section{Comparison with the Experiments of Doran}

In contrast to the experimental results reported above often a strong radial constriction of the Townsend discharge occurs during the development of the first luminous front. This was found by DorAN ${ }^{13}$, and still more clear in own experiments (Fig. 6). Doran explained it as a consequence of the finite radial extension of the space charge: The field distortion generated by the positive space charge has a maximum at the discharge axis and thus the succeeding avalanches get more amplification there. In a series of experiments it could however be shown that the real reason for the constriction was the following: When in a series of experiments the glow stage of previous discharges has developed till the spark stage - usually near the axis of the gap - a higher efficiency for secondary electron emission by photo effect is produced there on the cathode. By this way a higher carrier density near the axis leads to the constriction in the succeeding experiments. The experimental conditions for the discharges of Fig. 6 were the same as for those of Fig. 4 except that by using a bigger capacity more energy was released so that the discharges had ended in the spark stage. By carefully polishing the cathode and by limiting the energy input of preceding discharges the constriction could be avoided. The diameter $\mathrm{D}$ of the resulting broad discharge was $5-12 \mathrm{~cm}$, depending on the treatment of the cathode.

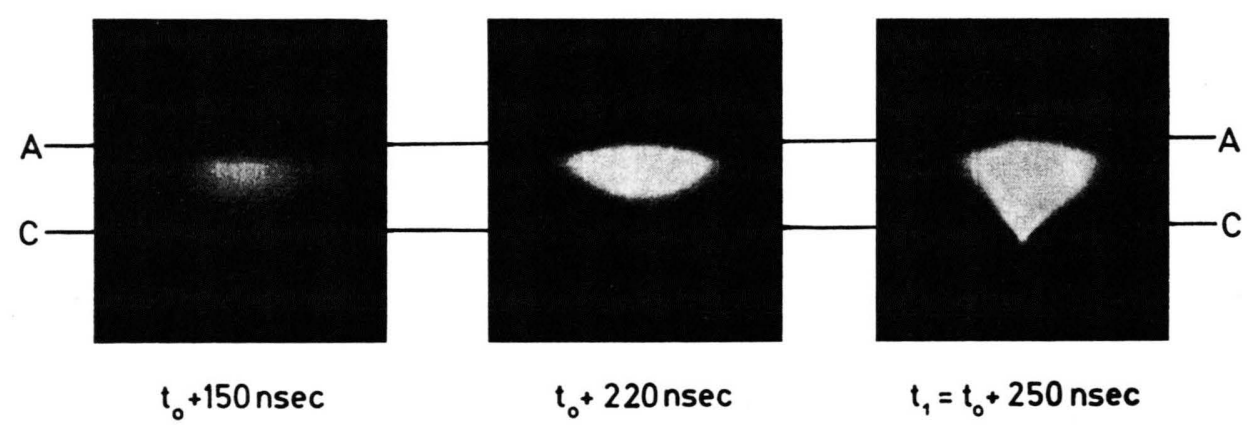

Fig. 6. Integrated shutter photographs (shutter closed at the time indicated) of a constricting Townsend discharge. Conditions and symbols as in Fig. 4. The series demonstrates how an initially laterally extended Townsend discharge constricts to a filamentary one under the influence of inhomogeneities in the electron emission of the cathode. 
The discharge current measured by DoRAN ${ }^{13}$ and the author for constricting discharges differs from that of the nonconstricting, see Fig. 5 a. This difference can be explained by the continuous reduction of the discharge area during the constriction, at least for the first stages when the discharge is not yet filamentary.

In streak photographs of the strongly constricting Townsend discharge a bright anode directed luminous front is visible between the first and second cathode directed wave front (Fig. 7). This is in contrast to Fig. 3 (one dimensional discharge). The different behaviour is certainly due to the very inhomogeneous field at the "tip" of the first

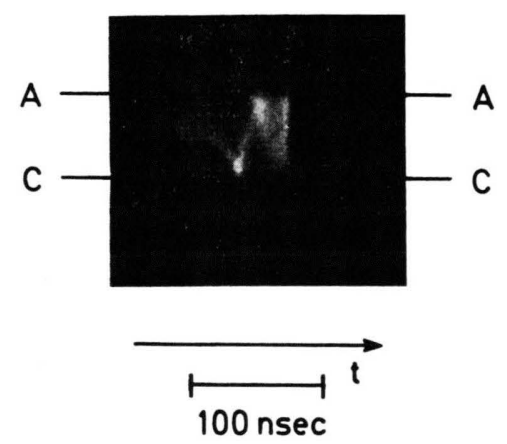

Fig. 7. Streak photograph of the constricting Townsend discharge of Fig. 6. Between the first and second cathode directed luminous front a very bright anode directed is visible in contrast to Fig. 3. It is a result of the constriction shown in Fig. 6. luminous front (see Fig. 6). The discharge is nearly filamentary (like a streamer discharge) after the time $t_{1}$, when the first luminous front arrives at the cathode. Thus the field distortions produced by the space charge in the wave front are high only near the wave front, while in the one dimensional discharge they are far reaching.

\section{Conclusion}

Fast luminous waves are found in the later stages of Townsend discharges (at low overvoltages) as well as in those of streamer discharges (at high overvoltages). For the streamer discharge new details of this development were derived from optical measurements with high time resolution. For Townsend discharges it could be demonstrated that at low overvoltages nearly one dimensional discharges without radial constriction are possible. The agreement between theoretical and experimental results for the stage of the luminous waves of these discharges is good. It could be shown how radial constrictions can be avoided.

I am indebted to Prof. Dr. H. Raether for many helpful discussions. The financial support of the Bundesministerium für Bildung und Wissenschaft is gratefully acknowledged. 